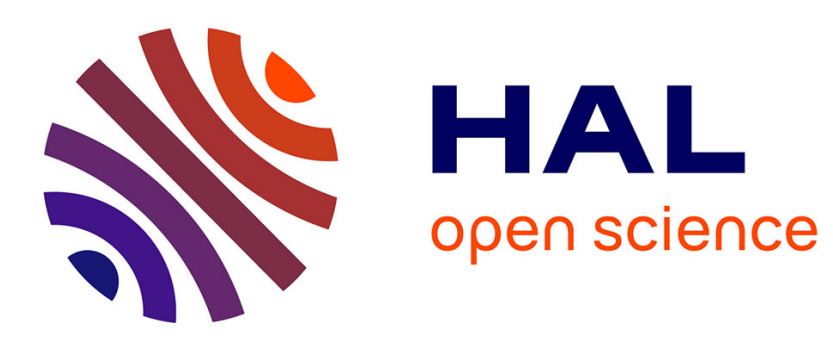

\title{
Voting with evaluations: characterizations of evaluative voting and range voting
}

\author{
Antonin Macé
}

\section{To cite this version:}

Antonin Macé. Voting with evaluations: characterizations of evaluative voting and range voting. 2017. halshs-01222200v2

\author{
HAL Id: halshs-01222200 \\ https://shs.hal.science/halshs-01222200v2 \\ Preprint submitted on 1 Feb 2018
}

HAL is a multi-disciplinary open access archive for the deposit and dissemination of scientific research documents, whether they are published or not. The documents may come from teaching and research institutions in France or abroad, or from public or private research centers.
L'archive ouverte pluridisciplinaire HAL, est destinée au dépôt et à la diffusion de documents scientifiques de niveau recherche, publiés ou non, émanant des établissements d'enseignement et de recherche français ou étrangers, des laboratoires publics ou privés. 


\title{
Voting with evaluations: characterizations of evaluative voting and range voting*
}

\author{
Antonin MACÉ
}

June 26, 2017

\begin{abstract}
I conduct an axiomatic analysis of voting rules in a context where voters evaluate each candidate by assigning her an evaluation from a pre-established set. I focus on additive rules, which follow the utilitarian paradigm. Characterization results are provided for each of the two prominent additive rules: Evaluative Voting when the evaluation set is finite and Range Voting when the evaluation set is $[0,1]$.
\end{abstract}

Keywords: Range Voting, Evaluative Voting, Utilitarianism, Measurement. JEL classification: D63, D70, C02

\footnotetext{
${ }^{*}$ I would like to thank Raicho Bojilov, Denis Bouyssou, Yukio Koriyama, Jean-François Laslier, François Maniquet, Vincent Merlin, Eduardo Perez, Robert Somogyi, Marjorie Sweetko and Rafael Treibich for useful remarks and comments, as well as participants in the economics seminars at École Polytechnique, ISI Delhi, the University of Warwick, the CORE, Maastricht University, the University of St. Andrews, the University of Manchester, Aix-Marseille School of Economics and in the following conferences: 4th Murat Sertel Workshop, ECORE 2013, Public Economic Theory 2013, Economic Design 2013, Economic Growth and Development 2013, RES 2014 and ADRES 2014. I am grateful to two reviewers and an editor for their helpful comments on a first version of the manuscript. All remaining errors are mine.

${ }^{\dagger}$ Aix-Marseille University (Aix-Marseille School of Economics), CNRS \& EHESS.
} 


\section{Introduction}

Most of the voting literature is set in the Arrovian framework, where voters rank the available candidates and Arrow's impossibility theorem prevails. I consider a different informational basis for social decisions, by allowing voters to evaluate candidates rather than to rank them. A celebrated example is the system of Approval Voting (Brams and Fishburn, 1978), in which two evaluations are available, "approve" and "disapprove", and the candidate with the most approvals wins the election. Extensions of this voting system with more than two evaluations have burgeoned recently in the literature and have even been experimented in the lab and in the field (Balinski and Laraki, 2011; Baujard et al., 2016). These extensions are appealing, as they offer a lot of freedom to voters, who can - to some extent - express the intensity of their preferences.

In a situation where voters evaluate candidates, the question arises of how best to aggregate evaluations so as to compare the candidates. Additive rules interpret the evaluations as grades, selecting as winner the candidate with the highest total grade. They are sometimes referred to as "utilitarian" voting rules (Hillinger, 2005). Other rules are conceivable as well, such as comparing candidates according to their median evaluation (Balinski and Laraki, 2011) or to their minimum evaluation (Aleskerov et al., 2010). To distinguish among existing rules, I provide an axiomatic analysis, with a focus on additive rules.

The first part of the analysis is set in a simple setting, where a rule is conceived as a ranking of evaluation vectors, that candidates may receive. Besides its application to voting procedures, the formal analysis is thus also relevant to ranking systems with evaluations. Such procedures are widely used in various settings, from the ranking of research projects by scientific committees to the ranking of restaurants or movies on designated websites.

Section 3 considers a discrete case where the number of available evaluations $\gamma$ is finite (Section 3). The Evaluative Voting rule assigns equally spaced values to the evaluations (from 1 to $\gamma$ ) and compares candidates according to their total value. I show that it is characterized by the axioms of Strong Pareto and Compensation - an increase in the evaluation of a voter can be compensated for by a decrease in the evaluation of another voter. I then provide a second 
and finer axiomatization of the rule, with a property of measurability of evaluation increments, which stresses the transparency of the rule.

Section 4 moves to the finer evaluation set [0,1] (Section 4). The counterpart of Evaluative Voting is then called Range Voting. In that case, imposing a measurability assumption is no longer necessary to obtain additive separability. First, applying Debreu's theorem, the class of additively separable rules is characterized with the help of an axiom of Separability - the evaluations of indifferent voters should not matter for social decisions. Second, I show that Range Voting is the only rule among this class that satisfies two natural invariance properties.

The aforementioned characterizations of Evaluative Voting and Range Voting are obtained in a simple setting of ranking rules. However, a voting rule can be best described, more generally, as a mapping from the profile of evaluation ballots to the winning candidate. To justify the use of the simple setting, Section 5 provides a structural result, describing conditions under which a voting rule is induced by a ranking rule. I conclude with the axiomatizations of Evaluative Voting and Range Voting in the general setting of voting rules.

The article contributes to the recent literature on "voting with evaluations" by providing a unified treatment of additive rules in that context. I show that Evaluative Voting can be characterized by a simple measurability assumption for arbitrary finite evaluation sets. This result is thus broader than the characterizations of Evaluative Voting obtained for three evaluations (Alcantud and Laruelle, 2013) and for numerical evaluations (Gaertner and Xu, 2012). In the continuous case, the axiomatization of Range Voting is more precise than that of Pivato (2013a), as it characterizes the rule within the class of additively separable rules, whereas Pivato (2013a) treats all rules of this class as equivalent to Range Voting. Furthermore, Section 6.1 uses the results of the discrete and continuous cases to compare the two related notions of Separability (for a fixed electorate) and Reinforcement (for a variable electorate), which have been shown to be central to the characterization of additively separable (resp. scoring) rules in the literature (Krantz et al., 1971; Pivato, 2013b). Finally, the structural result provided in Section 5, relating voting rules to ranking rules, is general and could be used to study other voting rules. 


\section{A simple framework : ranking rules}

The electorate is composed of a finite set of voters $I$. Voters vote by evaluating a finite set of candidates $X$. The evaluation set $\Gamma$ is a language through which voters express their opinion: they assign an evaluation in $\Gamma$ to each candidate. The set $\Gamma$ is endowed with a linear order $\geq_{\Gamma}$ (asymmetric part: $>_{\Gamma}$ ), where $a>_{\Gamma} b$ means that $a$ is a better evaluation than $b$. There is no restriction on the ballots - the set of ballots is $\Gamma^{X}$ - for instance, a voter can assign the same evaluation to several candidates.

I consider two types of evaluation sets in this article. The first case deals with a finite evaluation set $\Gamma$. This case is general, the evaluations can be numerical (e.g. $\Gamma=\{1,2,3\}$ ) or qualitative (e.g. $\Gamma=\{A, B, C\}$ ). In the second case, I take $\Gamma=[0,1]$, so that voters can express their opinion from a finer grid.

As each candidate receives an evaluation vector $u=\left(u_{i}\right)_{i \in I} \in \Gamma^{I}$ in this election, I define an aggregation rule as a ranking on evaluation vectors. ${ }^{1}$ The implicit assumption here is that the candidate whose evaluation vector is ranked the highest will be elected, this assumption will be later justified in Section 5. Formally, a ranking rule is a complete and transitive binary relation $\succeq$ on the set of evaluation vectors $\Gamma^{I}$. I denote by $\sim$ its symmetric part and by $\succ$ its asymmetric part.

Range Voting (RV). This rule was proposed by Smith (2000) for the evaluation set $\Gamma=$ $[0,1]$. The rule is additive: $u \succeq^{R V} v \Leftrightarrow \sum_{i \in I} u_{i} \geq \sum_{i \in I} v_{i}$. It is formally equivalent to the utilitarian social welfare ordering, but the evaluations are restricted to fall between 0 and 1 .

Evaluative Voting (EV). This rule, proposed by Hillinger (2005), is a discrete analog of Range Voting. I define it for any finite evaluation set $\Gamma$. Let $\gamma$ be the number of evaluations in $\Gamma: \gamma=\# \Gamma$. First, define the EV-value $f^{E V}: \Gamma \rightarrow\{1, \ldots, \gamma\}$ by $f^{E V}(a)=\#\left\{b \in \Gamma \mid a \geq_{\Gamma} b\right\}$. The EV-value of an evaluation $a \in \Gamma$ is the number of evaluations that are not better than $a .^{2}$

\footnotetext{
${ }^{1}$ This notion corresponds to the concept of social welfare ordering in the economic theories of justice (d'Aspremont and Gevers, 2002), the difference here being that $\Gamma \neq \mathbb{R}$.

${ }^{2}$ There is an immediate parallel with the Borda score for ordinal rankings: in that case, the score for being ranked at the $j$-th position is the number of positions not higher than $j$.
} 
Second, the Evaluative Voting rule is defined by $u \succeq^{E V} v \Leftrightarrow \sum_{i \in I} f^{E V}\left(u_{i}\right) \geq \sum_{i \in I} f^{E V}\left(v_{i}\right)$.

Two particular cases of EV are worth mentioning. When $\Gamma=\{$ "approve", "disapprove" $\}$, with the order "approve" $>_{\Gamma}$ "disapprove", EV is exactly the Approval Voting rule. Another noteworthy benchmark is when the number of evaluations equals the number of candidates: $\gamma=\# X$. There are two types of ballots in that case: those which assign each evaluation to exactly one candidate and those which do not. Each ballot of the former type is uniquely linked to an ordinal ranking on the set of candidates. If all ballots are of this type, it is easy to see that the candidate elected under EV is the Borda winner of the associated profile of rankings. Thus, when $\gamma=\# X, \mathrm{EV}$ can be seen as an extension of the Borda voting system, because voters are in no way restricted when assigning evaluations to candidates.

\section{Discrete scale: Evaluative Voting}

In this section, the evaluation set $\Gamma$ is finite, and I sometimes refer to the example where $\Gamma=\{A, B, C, D\}$ with $A>_{\Gamma} B>_{\Gamma} C>_{\Gamma} D$. For a given evaluation set $\Gamma$, what properties are characteristic of the EV rule?

The first remark is that the EV rule is efficient with respect to the order $\geq_{\Gamma}$ on the evaluations, as captured by the following property. A rule satisfies Strong Pareto (SP) if for any $u, v \in \Gamma^{I}$ such that $\exists i \in I,\left(u_{i}>_{\Gamma} v_{i}\right)$ and $\left(\forall j \neq i, u_{j} \geq_{\Gamma} v_{j}\right)$, we have $u \succ v$. Note that this axiom also conveys a notion of positive responsiveness (May, 1952) of the rule: when any of her evaluations is raised, a candidate must benefit.

Another distinctive feature of the EV rule is that it involves interpersonal measurability of evaluation increments. If $a \in \Gamma$ is not the highest evaluation in $\Gamma$, we denote by $S(a)$ the successor of $a$, formally: $S(a)=\min \left\{b \in \Gamma \mid b \succ_{\Gamma} a\right\}$. A rule $\succeq$ respects Compensation (Comp) if for any $u, v \in \Gamma^{I}$ such that $\exists i, j \in I,\left(v_{i}=S\left(u_{i}\right), u_{j}=S\left(v_{j}\right)\right)$ and $\left(\forall k \neq i, j, u_{k}=\right.$ $v_{k}$ ), we have $u \sim v$. This axiom states that, starting from any evaluation vector $u$, a singleincrement rise in the evaluation of a voter $i$ (e.g. from $C$ to $B$ ) can be compensated for by a single-increment fall in the evaluation of another voter $j$ (e.g. from $A$ to $B$ ). 
The axiom (Comp) follows the tradition of cancellation axioms, stating that a tie between the candidates should be declared for some "balanced" profiles of ballots. These axioms have been used to characterize the Borda rule (Young, 1974), the Approval Voting rule (Alós-Ferrer, 2006) and the EV rule in a specific case with three evaluations, when $\Gamma=\{$ "in favor", "indifferent", "against" $\}$ (Alcantud and Laruelle, 2013). ${ }^{3}$ The two former properties are sufficient to characterize the EV rule.

Theorem 1. Let $\Gamma$ be a finite set. A rule $\succeq$ satisfies (SP) and (Comp) if and only if it is the Evaluative Voting rule $\succeq^{E V}$.

This axiomatization of the EV rule holds for any finite evaluation set $\Gamma$ : no assumption is made on the number of evaluations, and the evaluations are only assumed to be linearly ordered. The result is in this sense more general than the characterizations of the EV rule provided in Alcantud and Laruelle (2013) for $\gamma=3$ and Gaertner and Xu (2012) for $\Gamma=\{1, \ldots, \gamma\}$. Moreover, the axiom (Comp) is weaker than the property of cancellation independence proposed by Gaertner and Xu (2012). ${ }^{4}$

Nevertheless, the property (Comp) may be considered as strong, as it contains both a requirement for anonymity between voters (voter $i$ is as important as voter $j$ ) and a requirement for measurability of evaluation increments (e.g. the distance from $B$ to $A$ is the same as from $C$ to $B)$. These two assumptions can be disentangled.

A rule respects Anonymity (A) if for any $u, v \in \Gamma^{I}$, for any permutation of the voters $\sigma: I \rightarrow I$, we have $u \sim u_{\sigma}$, where $u_{\sigma}=\left(u_{\sigma(i)}\right)_{i \in I}$. This is a natural property of fairness among voters. A rule is Independent of a Single-Increment Rise in the Evaluations (ISIRE) if for any $u, v, u^{\prime}, v^{\prime} \in \Gamma^{I}$ such that $\exists i \in I,\left(u_{i}^{\prime}=S\left(u_{i}\right), v_{i}^{\prime}=S\left(v_{i}\right)\right)$ and $\left(\forall k \neq i, u_{k}^{\prime}=u_{k}, v_{k}^{\prime}=v_{k}\right)$, we have $u \succeq v \Leftrightarrow u^{\prime} \succeq v^{\prime}$. In words, the ranking between two candidates is unaffected if a

\footnotetext{
${ }^{3}$ Alcantud and Laruelle (2013) show that cancellation (corresponding to axiom (Comp) here) can be weakened when faithfulness and consistency hold, applying only to profiles such that each candidate receives the same number of approvals and disapprovals. In my general setting with an arbitrary number of evaluations, such weakening is also feasible, but probably less interesting, as the weakened axiom would appear less canonical.

${ }^{4}$ As apparent from the proof, axiom (Comp) only requires the set $\left\{u \mid \sum_{i \in I} f^{E V}\left(u_{i}\right)=s\right\}$ to be contained in a social indifference curve, for each $s \in\{n, \ldots, n \gamma\}$. Contrastingly, cancellation independence in Gaertner and $\mathrm{Xu}$ (2012) implies that these sets are ranked monotonically according to $s$. Therefore, there are only three rules satisfying cancellation independence, whereas the number of rules satisfying (Comp) is the $(n \gamma-n+1)$-th ordered Bell number (number of orderings of $(n \gamma-n+1)$ elements, growing more than exponentially).
} 
voter $i$ increases her evaluation for each candidate by a single increment (e.g. from B to A for one candidate and from $\mathrm{C}$ to $\mathrm{B}$ for the other). From a practical perspective, this axiom is compelling: a rule satisfying (ISIRE) can be viewed as transparent, as any voter can easily understand that any increment has the same importance, in any situation.

Counter-example: the leximin rule violates (ISIRE). The leximin rule (d'Aspremont and Gevers, 2002) $\succeq^{\text {lex }}$ ranks $u$ higher than $v$ if the lowest evaluation in $u$ is higher than the lowest evaluation in $v$ (and iteratively so in case of equality). Consider the evaluation vectors $u=(A, D), v=(C, C), u^{\prime}=(A, C), v^{\prime}=(C, B)$. It is clear that $u \prec^{\text {lex }} v$ and $u^{\prime} \succ^{\text {lex }} v^{\prime}$, although the primed vectors only differ from the non-primed vectors because of the second voter, who raised her evaluations by a single increment in both vectors. In that case, the two increments weight differently (the one from $D$ to $C$ is more significant than the one from $C$ to $B)$.

These axioms lead to a second and more disaggregated characterization of the EV rule.

Theorem 2. Let $\Gamma$ be a finite set. A rule $\succeq$ satisfies (SP), (A) and (ISIRE) if and only if it is the Evaluative Voting rule $\succeq^{E V}$. Moreover, the three axioms are mutually independent.

The result pins down the main properties of EV: it is the only efficient and anonymous rule that is transparent, in the sense that it gives equal weight to all evaluation increments.

\section{Continuous scale: Range Voting}

In this section, we consider the continuous evaluation set $\Gamma=[0,1]$. For this case, the results obtained in the previous section can be directly adapted to characterize the Range Voting rule. However, we show that the continuous structure of the evaluation set allows to derive a characterization of the Range Voting rule without imposing any measurability requirement.

In fact, the axiom (ISIRE) can be considered as strong, as it contains both a requirement for measurability of evaluation increments and a requirement for separability. The axiom (ISIRE) 
refers to a situation where a voter $i$ increases all her evaluations by the same amount, ${ }^{5}$ and requires the social ranking to be unchanged - it should be the same between $u^{\prime}$ and $v^{\prime}$ and between $u$ and $v$. When $u_{i} \neq v_{i}$, this implies that increasing the evaluations by the same amount always has the same impact, whatever the original evaluation level (e.g. raising one's evaluation from 0.1 to 0.2 has the same effect as raising it from 0.8 to 0.9 ). When $u_{i}=v_{i}$, the fact that the ranking remains unchanged is a property in its own right, known as separability or independence of indifferent voters.

Formally, a rule satisfies Separability (Sep) if for any $u, v, u^{\prime}, v^{\prime} \in \Gamma^{I}$, for any subset of voters $J \subseteq I$ such that $\left(\forall j \in J, u_{j}=v_{j}, u_{j}^{\prime}=v_{j}^{\prime}\right)$ and $\left(\forall i \in I \backslash J, u_{i}^{\prime}=u_{i}, v_{i}^{\prime}=v_{i}\right)$, we have $u \succeq v \Leftrightarrow u^{\prime} \succeq v^{\prime}$. In this definition, voters in $J$ are indifferent between $u$ and $v$, and also between $u^{\prime}$ and $v^{\prime}$. The property conveys a notion of subsidiarity of the rule: to compare two candidates, we need not take into account the evaluations assigned by indifferent voters. Although natural, the axiom is violated by prominent rules, such as the median rule.

Counter-example: the median rule violates (Sep). For any odd number of voters, the median rule $\succeq^{\text {med }}$ ranks $u$ above $v$ if the median of $u$ is above the median of $v$ (Balinski and Laraki, 2011). With three voters, consider the evaluation vectors $u=(0.6,0.4,1), v=$ $(0.8,0.2,1), u^{\prime}=(0.6,0.4,0), v^{\prime}=(0.8,0.2,0)$. It is clear that $u \prec^{\text {med }} v$ and $u^{\prime} \succ^{\text {med }} v^{\prime}$, although the primed vectors only differ from the non-primed vectors by the evaluation of the third voter, who is indifferent between $u$ and $v$ and between $u^{\prime}$ and $v^{\prime}$.

In the sequel, the axiom (Sep) is used to characterize the class of additively separable rules, to which the Range Voting rule belongs. We say that a rule is continuous (Cont) if for any $u \in[0,1]^{I}$, the sets $\left\{v \in[0,1]^{I} \mid v \succeq u\right\}$ and $\left\{v \in[0,1]^{I} \mid u \succeq v\right\}$ are closed with respect to the Euclidean topology on $[0,1]^{I}$. This property expresses the natural view that small changes in the inputs of the rule - the evaluations - should not lead to dramatic changes in the output of the rule - the ranking among candidates' evaluation vectors. Applying Debreu's theorem, we

\footnotetext{
${ }^{5} \mathrm{~A}$ direct adaptation of (ISIRE) to the evaluation set $\Gamma=[0,1]$ would require independence for any transformation of the form $u_{i}^{\prime}=u_{i}+\lambda, v_{i}^{\prime}=v_{i}+\lambda$, with $\lambda>0$.
} 
obtain the following characterization of the class of additively separable rules. ${ }^{6}$

Theorem 3. Let $\Gamma=[0,1]$ and $\# I \geq 3$. A rule $\succeq$ satisfies (SP), (A), (Sep) and (Cont) if and only if it is additively separable: there exists a continuous and increasing function $f: \Gamma \rightarrow \mathbb{R}$ such that $\forall u, v \in \Gamma^{I}, u \succeq v \Leftrightarrow \sum_{i \in I} f\left(u_{i}\right) \geq \sum_{i \in I} f\left(v_{i}\right)$. Moreover, the four axioms are mutually independent.

Theorem 3 leaves some space for further exploration. An additively separable rule transforms the evaluations with some function $f$ before summing them, raising the question of which additional conditions are required for this function to be the identity. ${ }^{7}$ I introduce two axioms, adapted to the evaluation set $[0,1]$, which are then shown to single out the RV rule.

A rule satisfies Contraction Invariance (CI) if for any $u, v \in[0,1]^{I}$ and scalar $\alpha \in(0,1)$, we have $u \sim v \Rightarrow \alpha \cdot u \sim \alpha \cdot v$. According to this axiom, ${ }^{8}$ if two candidates are equally ranked by the rule, they should remain equally ranked after a contraction of all their evaluations by the same scalar $\alpha \in(0,1)$.

A rule respects Symmetry Invariance (SI) if for any $u, v \in[0,1]^{I}$, we have $u \sim v \Rightarrow$ $\mathbb{1}-u \sim \mathbb{1}-v$, where $\mathbb{1}-u=\left(1-u_{i}\right)_{i \in I}$. This axiom expresses the symmetry of the rule with respect to the evaluation set $[0,1] .^{9}$ With this set, the minimal evaluation that a voter can assign is 0 and the maximal evaluation is 1 . Thus, if candidate $x$ receives evaluation $a$ from a voter, this evaluation can be interpreted either as a vote by $a$ in favor of $x$ or as a vote by $(1-a)$ against $x$. According to this interpretation, the axiom (SI) requires the rule to treat symmetrically the votes in favor of a candidate and the votes against her. ${ }^{10}$

\footnotetext{
${ }^{6}$ In the additive conjoint measurement literature (Krantz et al., 1971), the proofs of such results are known to be long and involved. Here, Theorem 3 is derived from a result by Debreu (1960). In an earlier version of the article (Macé, 2015), a direct proof was provided.

${ }^{7}$ This approach has been taken by Maskin (1978) to single out the utilitarian social welfare ordering among the class of generalized utilitarianism, with the axiom of Cardinal Full Comparability. The axioms introduced here differ from that property, as the rules I consider are not defined on the same domain.

${ }^{8}$ In the literature on social welfare orderings, a similar property is captured by the axiom of Independence of the Common Utility Scale (Moulin, 1991).

${ }^{9}$ This axiom is reminiscent of the property of self-duality in the literature on the bankruptcy problem (Thomson, 2003).

${ }^{10}$ If $u$ is equivalent to $v$ in favor of a candidate, then (SI) implies that $u$ is equivalent to $v$ against a candidate, this means that $\mathbb{1}-v$ is equivalent to $\mathbb{1}-u$ in favor of a candidate.
} 
Theorem 4. Let $\Gamma=[0,1]$ and $\# I \geq 3$. A rule $\succeq$ satisfies (SP), (A), (Sep), (Cont), (CI) and (SI) if and only if it is the Range Voting rule $\succeq^{R V}$. Moreover, the six axioms are mutually independent.

This axiomatization of the RV rule differs from and complements the one provided in Pivato (2013a). In that article, RV is characterized up to an equivalence relation between rules, and this equivalence class corresponds to the class of additively separable rules in my framework. Thus, Theorem 4 goes beyond Pivato's axiomatization, as it explicitly takes into account the evaluation set $[0,1]$ and describes invariance properties that are characteristic of the RV rule, encoded in the axioms (CI) and (SI).

\section{A general framework: voting rules}

I focused in the previous sections on the simple setting of ranking rules, which compare evaluation vectors, that candidates may receive. In this section, I introduce a more natural and more general notion of aggregation rule and I provide characterization theorems of additive rules in this context.

\section{$5.1 \quad$ Voting rules}

The input of a voting rule is an evaluation profile, a matrix $m=\left(m_{i}^{x}\right)_{i \in I}^{x \in X}$, with $m_{i}=\left(m_{i}^{x}\right)^{x \in X} \in$ $\Gamma^{X}$ being the ballot cast by voter $i$ and $m^{x}=\left(m_{i}^{x}\right)_{i \in I} \in \Gamma^{I}$ being the evaluation vector received by candidate $x$. I denote by $M=\Gamma^{X \times I}$ the set of all evaluation profiles. A voting rule is a correspondence $\Phi: M \rightrightarrows X$ such that for any profile $m \in M, \Phi(m) \neq \emptyset$. The set $\Phi(m)$ is the set of winning candidates, it is typically a singleton but it may contain several candidates if there is a tie. ${ }^{11}$ This notion of a voting rule directly coincides with the procedure used to aggregate ballots in a voting system with evaluations, and it corresponds to the definition used in the literature (for instance in Pivato (2013a); Alcantud and Laruelle (2013)). We say that a voting rule $\Phi$ is represented by a ranking rule $\succeq$ if $\forall m \in M, \Phi(m)=\left\{x \in X \mid \forall y \in X, m^{x} \succeq m^{y}\right\}$. It

\footnotetext{
${ }^{11}$ Ties are assumed to be broken randomly.
} 
follows that every ranking rule induces a voting rule, and I denote by $\Phi^{E V}$ and $\Phi^{R V}$ the voting rules induced by $\succeq^{E V}$ and $\succeq^{R V}$ respectively. As we shall see below, some voting rules are not represented by a ranking rule.

\section{$5.2 \quad$ A representation theorem}

I introduce two axioms which are satisfied when a voting rule is represented by a ranking rule. First, a rule $\Phi$ is neutral $\left(\mathbf{N}^{*}\right)$ if for any permutation $\sigma$ of $X$ and any profile $m \in M$, we have $x \in \Phi(m) \Leftrightarrow \sigma(x) \in \Phi\left(m^{\sigma}\right)$, where $m^{\sigma}=\left(m^{\sigma(x)}\right)^{x \in X}$. A neutral rule does not depend on the names of the candidates. Second, a rule satisfies Binary Independence (BI*) if for all profiles $m, n \in M$, for all candidates $x, y \in X$ such that $m^{x}=n^{x}$ and $m^{y}=n^{y}$, we have $x \in \Phi(m) \Rightarrow(y \in \Phi(n) \Rightarrow x \in \Phi(n))$. In words, if $m^{x}$ is revealed to be (weakly) preferred to $m^{y}$ in profile $m$, this should remain true in profile $n$, although the evaluations attributed to other candidates than $x$ and $y$ may have changed.

Counter-example: the relative utilitarian rule violates $\left(\mathrm{BI}^{*}\right)$. For $\Gamma=[0,1]$, the relative utilitarian rule ${ }^{12}$ (Dhillon and Mertens, 1999) is defined by

$$
\Phi^{R U}(m)=\arg \max _{x \in X} \sum_{i \in I} g^{x}\left(m_{i}\right), \quad \text { with } g^{x}\left(m_{i}\right)=\frac{m_{i}^{x}-\min _{y \in X} m_{i}^{y}}{\max _{y \in X} m_{i}^{y}-\min _{y \in X} m_{i}^{y}} \cdot{ }^{13}
$$

This rule re-scales each evaluation $m_{i}^{x}$, so that $i$ 's preferred candidate receives the value 1 and $i$ 's least preferred candidate receives the value 0 . As the worth of a candidate depends on the evaluations received by other candidates, it is easy to see that the rule $\Phi^{R U}$ violates (BI*).

The axioms $\left(\mathrm{N}^{*}\right)$ and $\left(\mathrm{BI}^{*}\right)$ have been consistently defended in the literature on voting rules, and as I show below, they jointly characterize the class of ranking rules.

Theorem 5. Let $\# X \geq 3$. A voting rule $\Phi$ satisfies axioms $\left(\boldsymbol{N}^{*}\right)$ and $\left(\boldsymbol{B I ^ { * } )}\right.$ if and only if it is represented by a ranking rule $\succeq$.

\footnotetext{
${ }^{12}$ The rule $\Phi^{R U}$ defined here is introduced as a benchmark, rather than a desirable rule. Whereas it is legitimate to re-scale individual utilities when there exists no common utility scale, it does not seem appropriate to re-scale the evaluations here, as voters have a common scale of evaluations $\Gamma$.

${ }^{13}$ by convention $\frac{0}{0}=0$.
} 
This is a structural result, providing conditions under which the choice between candidates can be resumed to a comparison of their evaluation vectors. ${ }^{14}$ Theorem 5 naturally relates to other results obtained in this vein, providing conditions under which a ranking of the candidates can be reduced to a ranking of their attributes (d'Aspremont and Gevers, 2002; Sprumont, 2016).

In the sequel, Theorem 5 is used to characterize EV and RV in the setting of voting rules. Axioms from the previous sections are adapted to this new setting and marked with a star.

\subsection{Discrete scale: Evaluative Voting}

A voting rule $\Phi$ is anonymous ( $\left.\mathbf{A}^{*}\right)$ if for any profile $m \in M$ and permutation $\sigma$ of $I$, we have $\Phi\left(m_{\sigma}\right)=\Phi(m)$, where $m_{\sigma}=\left(m_{\sigma(i)}\right)_{i \in I}$. A rule $\Phi$ satisfies Strong Pareto (SP*) if for any profile $m \in M$ and candidate $x \in X$ such that for all other candidate $z,\left(\forall i \in I, m_{i}^{z} \geq_{\Gamma} m_{i}^{x}\right)$ and $\left(\exists j \in I, m_{j}^{z}>_{\Gamma} m_{j}^{x}\right)$, we have $x \notin \Phi(m)$. In words, if a candidate $x$ is strictly Pareto-dominated by all other candidates, she should not be elected. When $\Gamma$ is finite, we say that a rule $\Phi$ is Independent of a Single-Increment Rise in the Evaluations (ISIRE*) if for any profiles $m, n \in M$ and voter $i \in I$ such that $\left(\forall x \in X, n_{i}^{x}=S\left(m_{i}^{x}\right)\right)$ and $\left(\forall j \neq i, n_{j}=m_{j}\right)$, we have $\Phi(m)=\Phi(n)$. As a corollary of Theorem 2 and Theorem 5, we obtain a characterization of the EV rule in the general setting.

Theorem 6. Let $\Gamma$ be a finite set and $\# X \geq 3$. A voting rule $\Phi$ satisfies $\left(\boldsymbol{N}^{*}\right),\left(\boldsymbol{B I ^ { * }}\right),\left(\boldsymbol{A}^{*}\right)$, $\left(\boldsymbol{S P}^{*}\right)$ and (ISIRE*) if and only if it is the Evaluative Voting rule $\Phi^{E V}$. Moreover, the five axioms are mutually independent.

\footnotetext{
${ }^{14}$ Note in the proof the generality of the result, which remains true if the attributes of the candidates lie in any abstract set (other than $\Gamma^{I}$ ). Besides, as we show later in the proof of Proposition 1 , the result remains true if we replace $\left(\mathrm{N}^{*}\right)$ by a weak neutrality axiom, requiring the full set of candidates to be elected when all candidates receive the same evaluation vector.
} 


\subsection{Continuous scale: Range Voting}

A voting rule $\Phi$ is continuous (Cont*) if for any candidate $x \in X$, the set $\{m \in M \mid x \in \Phi(m)\}$ is closed with respect to the product topology on $M=\Gamma^{X \times I} \cdot{ }^{15}$ Quite surprisingly, we observe that the axiom of neutrality can be obtained as a by-product of the axioms $\left(\mathrm{BI}^{*}\right),\left(\mathrm{SP}^{*}\right)$ and (Cont*) when the evaluation set is continuous.

Proposition 1. Let $\Gamma=[0,1]$ and $\# X \geq 3$. If a voting rule $\Phi$ satisfies axioms $\left(\boldsymbol{B I}^{*}\right),\left(\boldsymbol{S P}^{*}\right)$ and $\left(\right.$ Cont $\left.^{*}\right)$, then it satisfies neutrality $\left(\boldsymbol{N}^{*}\right)$.

The proof of Proposition 1 starts with the observation that (SP*) and (Cont*) together imply a weak neutrality property, requiring the full set of candidates to be elected when all candidates receive the same evaluation vector. The bulk of the proof then consists in showing that this property, together with $\left(\mathrm{BI}^{*}\right)$, implies the stronger axiom $\left(\mathrm{N}^{*}\right)$.

A rule $\Phi$ is separable (Sep*) if for all profiles $m, n \in M$ and subset of voters $J \subseteq I$ such that $\left(\forall i \in J, \forall x, y \in X, m_{i}^{x}=m_{i}^{y}, n_{i}^{x}=n_{i}^{y}\right)$ and $\left(\forall i \in I \backslash J, m_{i}=n_{i}\right)$, we have $\Phi(m)=\Phi(n)$. For $\Gamma=[0,1]$, a rule $\Phi$ satisfies Contraction Invariance (CI*) if for any profile $m \in M$, candidate $x \in X$ and scalar $\alpha \in(0,1)$, we have $\Phi(m)=X \Rightarrow \Phi(\alpha \cdot m)=X$. A rule $\Phi$ satisfies Symmetry Invariance (SI*) if for any profile $m \in M, \Phi(m)=X \Rightarrow \Phi(\mathbb{1}-m)=X$, where $\mathbb{1}-m=\left(1-m_{i}^{x}\right)_{i \in I}^{x \in X}$. In this general setting, the axioms $\left(\mathrm{CI}^{*}\right)$ and $\left(\mathrm{SI}^{*}\right)$ impose weak invariance requirements: if all the candidates are chosen in one profile, then they should also be all chosen in the modified profile. As a corollary of Theorem 4, Theorem 5 and Proposition 1, we obtain a characterization of the RV rule in the general setting.

Theorem 7. Let $\Gamma=[0,1], \# X \geq 3$ and $\# I \geq 3$. A voting rule $\Phi$ satisfies $\left(\boldsymbol{B I}^{*}\right)$, $\left(\boldsymbol{A}^{*}\right)$, $\left(\boldsymbol{S P}^{*}\right),\left(\boldsymbol{C o n t}^{*}\right),\left(\boldsymbol{S e p}^{*}\right),\left(\boldsymbol{C I}^{*}\right)$ and $\left(\boldsymbol{S I}^{*}\right)$ if and only if it is the Range Voting rule $\Phi^{R V}$. Moreover, the seven axioms are mutually independent.

Note that, by virtue of Proposition 1, axiom $\left(\mathrm{N}^{*}\right)$ need not be invoked, and the characterization is obtained with independent axioms.

\footnotetext{
${ }^{15}$ In the literature, this notion is sometimes referred to as the hemicontinuity of the correspondence $\Phi$ (see Ok (2007)).
} 


\section{Concluding remarks}

\subsection{Discrete scale versus continuous scale}

Section 4 shows how the class of additively separable rules can be characterized with the axiom (Sep) when the evaluation set is $[0,1]$. The following counter-example demonstrates that this characterization does not extend for a discrete scale, ${ }^{16}$ and suggests that Theorem 4 and Theorem 7 cannot be adapted to axiomatize the EV rule.

Proposition 2. For $\# I=3$ and $\gamma=4$, there exists a non-additively separable rule satisfying (SP), (A) and (Sep).

This result also reveals a distinction between the axiom of Separability, defined for a fixed electorate, and the axiom of Reinforcement ${ }^{17}$ defined for a variable electorate. The latter axiom has often been used to characterize the class of scoring rules, to which EV and RV belong, under various assumptions on the nature of the ballots (Smith, 1973; Young, 1975; Myerson, 1995; Balinski and Laraki, 2011; Pivato, 2013b). Although both axioms convey a similar notion of consistency of collective decisions, the axiom of Reinforcement is formally stronger than the axiom of Separability. On the one hand, it is easy to see that a variable-population rule satisfying Reinforcement is separable for any fixed electorate. On the other hand, there are separable rules defined for a fixed electorate that cannot be extended to a (anonymous) variable-electorate rule satisfying Reinforcement, such as the rule considered in the proof of Proposition 2.

\subsection{Strategic properties of EV and RV rules}

The analysis presented in this article is rooted in the assumption that voters truthfully evaluate the candidates, which could be called into question. If the election is conceived as a process of preference aggregation, Núñez and Laslier (2014) have shown that the outcome of an election

\footnotetext{
${ }^{16}$ The literature (Krantz et al., 1971) identifies a countable family of axioms, extending (Sep), that are necessary to have an additively separable rule, for arbitrary $\# I$ and $\gamma$. The counter-example is thus general. In a previous version of the paper (Macé, 2015), I pointed out that (SP), (A) and (Sep) characterize additive separability in the specific case where $\gamma \leq 3$.

${ }^{17}$ This axiom states that if two sub-electorates agree on a ranking between two candidates, then the rule applied to the joint electorate should produce the same ranking.
} 
under the EV rule is the same as under Approval Voting, provided that the electorate is large and strategic. In this perspective, it is just as if intermediate evaluations do not matter. Nevertheless, if voters' expressive motives are stronger than their strategic motives, one might expect voters to make use of the full spectrum of evaluations in their ballots, ${ }^{18}$ and this could lead to collective judgments more aligned with the utilitarian principle than those obtained under Approval Voting. Finally, if the election is conceived as a process of information aggregation, the flexibility of the EV (or RV) rule allows for a nuanced and rich information revelation by voters, which could improve the quality of information aggregation compared to other voting rules. This last consideration could be explored in more detail in further research.

\section{A Appendix}

\section{A.1 Proof of Theorem 1}

It is straightforward that EV satisfies all the mentioned axioms. Let $\succeq$ be a rule satisfying axioms (SP) and (Comp). Let $u, v \in \Gamma^{I}$ be two evaluation vectors such that $\sum_{i \in I} f^{E V}\left(u_{i}\right)=$ $\sum_{i \in I} f^{E V}\left(v_{i}\right)$. The vector $v$ can be obtained from $u$ by a finite sequence of transformations of the form $\left\{\left(u_{i}, u_{j}\right) \mapsto\left(S\left(u_{i}\right), P\left(u_{j}\right)\right)\right\}$, where $P(a)$ denotes the predecessor of evaluation $a$ $(b=P(a)$ if and only if $a=S(b))$. It follows, by iterated application of axiom (Comp) and transitivity of $\sim$, that $u \sim v$.

As a result, there exists a function $F:\{n, \ldots, n \gamma\} \rightarrow \mathbb{R}$ such that:

$$
u \succeq v \quad \Leftrightarrow \quad F\left(\sum_{i \in I} f^{E V}\left(u_{i}\right)\right) \geq F\left(\sum_{i \in I} f^{E V}\left(v_{i}\right)\right)
$$

By axiom (SP), the function $F$ is increasing. Finally, the rule $\succeq$ is the EV rule.

\footnotetext{
${ }^{18}$ This is indeed what has been observed in experiments conducted on the EV rule, and in particular for in situ experiments, in which voters have actual political preferences (Igersheim et al., 2016).
} 


\section{A.2 Proof of Theorem 2}

It is straightforward that EV satisfies all the mentioned axioms. In the sequel, I show that (A) and (ISIRE) imply together (Comp), and the result becomes a corollary of Theorem 1 . Let $\succeq$ be a rule satisfying axioms (A) and (ISIRE). Let $u$ and $v$ be two evaluation vectors such that $v_{i}=S\left(u_{i}\right), u_{j}=S\left(v_{j}\right)$ and $\forall k \neq i, j, v_{k}=u_{k}$. I note $u_{-i, j}=\left(u_{k}\right)_{k \neq i, j}$ and obtain successively:

$$
\begin{aligned}
\left(u_{i}, v_{j}, u_{-i, j}\right) & \sim\left(v_{j}, u_{i}, u_{-i, j}\right) \\
\left(u_{i}, S\left(v_{j}\right), u_{-i, j}\right) & \sim\left(v_{j}, S\left(u_{i}\right), u_{-i, j}\right) \\
\left(u_{i}, u_{j}, u_{-i, j}\right) & \sim\left(v_{j}, v_{i}, u_{-i, j}\right) \\
\left(u_{i}, u_{j}, u_{-i, j}\right) & \sim\left(v_{i}, v_{j}, u_{-i, j}\right) \\
u & \sim v
\end{aligned}
$$

Therefore, the rule $\succeq$ satisfies (Comp).

Independence of the axioms. Axiom (A) is violated by the rule: $u \succeq v \Leftrightarrow \sum_{i \in I} \lambda_{i} f^{E V}\left(u_{i}\right) \geq$ $\sum_{i \in I} \lambda_{i} f^{E V}\left(v_{i}\right)$ if the weights $\left(\lambda_{i}\right)_{i \in I}$ are not all equal. Axiom (SP) is not satisfied by the constant rule (for which all vectors are equivalent). Finally, axiom (ISIRE) is violated by the leximin rule (d'Aspremont and Gevers, 2002).

\section{A.3 Proof of Theorem 3}

It is clear that RV satisfies all the mentioned axioms. Let $\succeq$ be a rule satisfying axioms (SP), (A), (Sep) and (Cont). I apply the result of Debreu (1960) on additive separability. As Debreu puts it, the relation $\succeq$ is a complete and continuous preordering on $[0,1]^{I}$, the product of $n$ connected and separable spaces. By axiom (Sep), the $n$ factors of $[0,1]^{I}$ are independent. By axiom (SP), each factor is essential. By application of Debreu's theorem, there exist continuous functions $f^{i}:[0,1] \rightarrow \mathbb{R}$ such that $u \succeq v \Leftrightarrow \sum_{i \in I} f^{i}\left(u_{i}\right) \geq \sum_{i \in I} f^{i}\left(v_{i}\right)$. By axiom (A), all the functions should be the same, $\forall i \in I, f^{i}=f$. By axiom (SP), the function $f$ is increasing. Therefore, the rule is additively separable. The independence of the axioms is a direct consequence of the 
independence of the axioms in Theorem 4, demonstrated below.

\section{A.4 Proof of Theorem 4}

It is clear that RV satisfies all the mentioned axioms. Let $\succeq$ be a rule satisfying axioms (SP), (A), (Sep), (Cont), (CI) and (SI). We know from Theorem 3 that there exists a continuous and increasing function $f:[0,1] \rightarrow \mathbb{R}$ such that $u \succeq v$ if and only if $\sum_{i \in I} f\left(u_{i}\right) \geq \sum_{i \in I} f\left(v_{i}\right)$. Let us assume without loss of generality that $f([0,1])=[0,1]$. I show that $\forall x \in[0,1], f(x)=x$.

Step 1. $\mathbf{f}(\mathrm{x})+\mathbf{f}(1-\mathbf{x})=1$ for all $\mathrm{x} \in[0,1]$.

Let $x \in[0,1]$ and define $z \in[0,1]$ as the unique number such that $f(z)=\frac{f(x)+f(1-x)}{2}$. We obtain $(z, z) \sim(x, 1-x)$. It follows from axioms $(\mathrm{SI})$ and $(\mathrm{A})$ that we have $(1-z, 1-z) \sim$ $(1-x, x) \sim(x, 1-x) \sim(z, z)$. An application of axiom (SP) gives $z=1-z$ and thus $z=\frac{1}{2}$. Finally we obtain that for all $x \in[0,1], f\left(\frac{1}{2}\right)=\frac{f(x)+f(1-x)}{2}$. Taking $x=0$ and knowing that $f(0)=0$ and $f(1)=1$, we find that $f\left(\frac{1}{2}\right)=\frac{1}{2}$. Thus: $\forall x \in[0,1], f(x)+f(1-x)=1$.

Step 2. $\mathbf{f}(\mathbf{x})=\mathbf{x}$ for any dyadic number $x \in[0,1]$.

For any integer $p \geq 1$, let us define $D_{p}=\left\{\frac{k}{2^{p}} \mid 0 \leq k \leq 2^{p}\right\}$. A number $x \in[0,1]$ is said to be dyadic if it belongs to some $D_{p}, p \geq 1$. I show by induction on $p$ that $f(x)=x$ for all $x \in D_{p}$. We know that $f(x)=x$ for all $x \in D_{1}$. Let $p \geq 2$ and assume that $f$ is the identity function on the set $D_{p-1}$. Let $d \in D_{p}: d$ can be written as $d=\frac{k}{2^{p}}$ with $k \in\left\{0, \ldots, 2^{p}\right\}$. If $k$ is even, we know that $d \in D_{p-1}$ and thus $f(d)=d$. Let us first consider the case where $k$ is odd and $k<2^{p-1}$, so that $d<\frac{1}{2}$.

We have $\left\{\frac{k-1}{2^{p-1}}, \frac{k}{2^{p-1}}, \frac{k+1}{2^{p-1}}\right\} \subseteq D_{p-1}$. It follows that $f\left(\frac{k-1}{2^{p-1}}\right)=\frac{k-1}{2^{p-1}}, f\left(\frac{k}{2^{p-1}}\right)=\frac{k}{2^{p-1}}$, $f\left(\frac{k+1}{2^{p-1}}\right)=\frac{k+1}{2^{p-1}}$. Thus, $\left(\frac{k}{2^{p-1}}, \frac{k}{2^{p-1}}\right) \sim\left(\frac{k-1}{2^{p-1}}, \frac{k+1}{2^{p-1}}\right)$.

Applying the axiom $(\mathrm{CI})$ with scalar $\alpha=\frac{1}{2}$, we get $\left(\frac{k}{2^{p}}, \frac{k}{2^{p}}\right) \sim\left(\frac{k-1}{2^{p}}, \frac{k+1}{2^{p}}\right)$. Moreover, since $k$ is odd, $k-1$ and $k+1$ are even, meaning that $\left\{\frac{k-1}{2^{p}}, \frac{k+1}{2^{p}}\right\} \subseteq D_{p-1}$. So, we know that $f\left(\frac{k-1}{2^{p}}\right)=\frac{k-1}{2^{p}}, \quad f\left(\frac{k+1}{2^{p}}\right)=\frac{k+1}{2^{p}}$. As a conclusion, we obtain $2 \times f\left(\frac{k}{2^{p}}\right)=\frac{k-1}{2^{p}}+\frac{k+1}{2^{p}}=2 \times \frac{k}{2^{p}}$. Finally $f(d)=d$. If $d>\frac{1}{2}$, we obtain $f(d)=d$ by application of Step 1 .

Conclusion : $\mathbf{f}(\mathbf{x})=\mathbf{x}$ for all $\mathbf{x} \in[\mathbf{0}, \mathbf{1}]$. This follows immediately from the continuity of $f$. Thus, the rule $\succeq$ is the RV rule. 
Independence of the axioms. I exhibit six different rules, each satisfying exactly five of the six axioms:

- a weighted utilitarianism $\succeq^{W U, \lambda}$ (d'Aspremont and Gevers, 2002), defined by $u \succeq^{W U, \lambda} v$ if $\sum_{i \in I} \lambda_{i} u_{i} \geq \sum_{i \in I} \lambda_{i} v_{i}$ for some vector $\left(\lambda_{i}\right)_{i \in I}$ of positive weights. If the weights are not all equal, $\succeq^{W U, \lambda}$ violates $(\mathrm{A})$. By linearity, all the other axioms are satisfied.

- the constant rule $\succeq^{C T}$, defined by $u \succeq^{C T} v$ for all $u, v \in[0,1]^{I}$, violates (SP).

- the leximin rule $\succeq^{L E X}$, (d'Aspremont and Gevers, 2002) violates (Cont). It satisfies the other axioms, in particular axiom (SI) as $u \sim^{L E X} v$ if and only if $u$ is a permutation of $v$.

- a weighted rank-utilitarianism $\succeq^{W R U, \lambda}$ (d'Aspremont and Gevers, 2002), defined by $u \succeq^{W R U, \lambda}$ $v$ if $\sum_{i \in I} \lambda_{i} \tilde{u}_{i} \geq \sum_{i \in I} \lambda_{i} \tilde{v}_{i}$ where $\left(\lambda_{i}\right)_{i \in I}$ is a vector of positive weights and $\tilde{u}$ (resp. $\tilde{v}$ ) denotes the sorted permutation of $u$ (resp $v$ ). If the weights are such that $\forall i \in I, \lambda_{i}=\lambda_{n-i}$ and are not all equal, the rule $\succeq^{W R U, \lambda}$ violates (Sep). It satisfies the other axioms, in particular axiom $(\mathrm{SI})$ as $\sum_{i \in I} \lambda_{i}(\widetilde{\mathbb{1}-u})_{i}=\sum_{i \in I} \lambda_{i}\left(1-\tilde{u}_{n-i}\right)=\left(\sum_{i \in I} \lambda_{i}\right)-\sum_{i \in I} \lambda_{i} \tilde{u}_{i}$.

- a symmetric rule $\succeq^{S Y M}$, defined by $u \succeq^{S Y M} v$ if $\sum_{i \in I} f\left(u_{i}\right) \geq \sum_{i \in I} f\left(v_{i}\right)$ with $f(x)=2 x^{2}$ if $x \leq \frac{1}{2}$ and $f(x)=1-2(1-x)^{2}$ if $x>\frac{1}{2}$. It violates $(\mathrm{CI})$ as $2 f(1 / 2)=f(0)+f(1)$, but $2 f(1 / 4)<f(0)+f(1 / 2)$. The rule satisfies the other axioms, in particular axiom (SI) as $f(1-x)=1-f(x)$ for all $x \in[0,1]$.

- the square rule $\succeq^{S Q}$, defined by $u \succeq^{S Q} v$ if $\sum_{i \in I} u_{i}^{2} \geq \sum_{i \in I} v_{i}^{2}$, violates (SI).

\section{A.5 Proof of Theorem 5}

It is immediate that if a voting rule is represented by a ranking rule, it satisfies both $\left(\mathbf{N}^{*}\right)$ and $\left(\mathbf{B I}^{*}\right)$. Now, let $\Phi$ be a voting rule satisfying $\left(\mathbf{N}^{*}\right)$ and $\left(\mathbf{B I}^{*}\right)$.

We define the binary relation $\succeq^{\Phi}$ on $\Gamma^{I}$ by $u \succeq^{\Phi} v$ if there exist a profile $m \in M$ and two candidates $x, y \in X$ such that $m^{x}=u, m^{y}=v$ and $x \in \Phi(m)$. Let us show that $\succeq^{\Phi}$ is a ranking rule. 
First, the relation $\succeq^{\Phi}$ is complete. Take $u, v \in \Gamma^{I}$, and let $x \in X$. Consider the profile $m$ defined by $m^{x}=u$ and $\forall y \neq x, m^{y}=v$. By definition of a voting rule, there must be at least one element in $\Phi(m)$. Thus, by definition of the relation $\succeq^{\Phi}$, we get either $u \succeq^{\Phi} v$ or $v \succeq^{\Phi} u$.

Second, the relation $\succeq^{\Phi}$ is transitive. Assume $u \succeq^{\Phi} v$ and $v \succeq^{\Phi} w$. There exists a profile $m \in M$, two candidates $x, y \in X$ such that $m^{x}=u, m^{y}=v$ and $x \in \Phi(m)$. There also exists a profile $n \in M$, two candidates $z, t \in X$ such that $m^{z}=v, m^{t}=w$ and $z \in \Phi(n)$. By neutrality $\left(\mathbf{N}^{*}\right)$ applied to the profile $n$, we can assume that $z=y$ and $t \neq x, y$. We construct a third profile $l$, defined by $l^{x}=u, l^{y}=v, l^{t}=w$ and for all $r \neq x, y, t, l^{r}=w$. We have three cases:

- if $x \in \Phi(l)$, then we obtain that $u \succeq^{\Phi} w$.

- if $y \in \Phi(l)$, then we apply binary independence (BI*): we have $x \in \Phi(m)$ and $y \in \Phi(l)$, with $m^{x}=l^{x}(=u)$ and $m^{y}=l^{y}(=v)$, therefore $x \in \Phi(l)$ and we are back to the first case.

- if $r \in \Phi(l)$ with $r \neq x, y$, we can assume by neutrality $\left(\mathbf{N}^{*}\right)$ that $r=t$ (remember that $\left.l^{r}=l^{t}=w\right)$. We apply binary independence $\left(\mathbf{B I}^{*}\right)$ : we have $y \in \Phi(n)$ and $t \in \Phi(l)$, with $n^{y}=l^{y}(=v)$ and $n^{t}=l^{t}(=w)$, therefore $y \in \Phi(l)$ and we are back to the second case.

Finally, we obtain that $u \succeq^{\Phi} w$ : the relation $\succeq^{\Phi}$ is transitive.

The final step is to show that the voting rule $\Phi$ is represented by the ranking rule $\succeq^{\Phi}$. First, if $x \in \Phi(m)$, we have by definition of $\succeq^{\Phi}: \forall y \in X, m^{x} \succeq^{\Phi} m^{y}$. Second, let $m \in M, x \in X$ be such that $\forall y \in X, m^{x} \succeq^{\Phi} m^{y}$. Let $y \in \Phi(m)$. We have by assumption $m^{x} \succeq^{\Phi} m^{y}$. This relation is obtained for some profile $n \in M$ and candidates $z, t \in X$, such that $n^{z}=m^{x}, n^{t}=m^{y}$ and $z \in \Phi(n)$. By neutrality $\left(\mathbf{N}^{*}\right)$, we may assume that $z=x$ and $t=y$. By application of binary independence (BI*), as we have $x \in \Phi(n)$ and $y \in \Phi(m)$, we get $x \in \Phi(m)$. Finally, $\Phi$ is represented by the ranking rule $\succeq^{\Phi}$.

Independence of the axioms. A rule electing always the same candidate satisfies (BI*) but not $\left(\mathbf{N}^{*}\right)$. Conversely, the rule $\Phi^{R U}$ satisfies $\left(\mathbf{N}^{*}\right)$ but not $\left(\mathbf{B I}^{*}\right)$. 


\section{A.6 Theorem 6: independence of the axioms}

Take a linear order $>_{X}$ on $X$. The rule $\Phi^{1}$ defined by

$$
\Phi^{1}(m)=\max \left\{x \in X \mid \forall y \in X, \sum_{i \in I} f^{E V}\left(m_{i}^{x}\right) \geq \sum_{i \in I} f^{E V}\left(m_{i}^{y}\right)\right\}
$$

satisfies axioms $\left(\mathbf{B I}^{*}\right),\left(\mathbf{A}^{*}\right),\left(\mathbf{S P}^{*}\right)$ and $\left.(\mathbf{I S I R E})^{*}\right)$ but not $\left(\mathbf{N}^{*}\right)$. Conversely, consider the discrete analog of the relative utilitarian rule, defined by $\Phi^{2}(m)=\arg \max _{x \in X} \sum_{i \in I} g^{x}\left(f^{E V}\left(m_{i}\right)\right)$. The rule $\Phi^{2}$ satisfies axioms $\left(\mathbf{N}^{*}\right),\left(\mathbf{A}^{*}\right),\left(\mathbf{S P}^{*}\right)$ and $\left(\mathbf{I S I R E} \mathbf{E}^{*}\right)$ but not $\left(\mathbf{B I}^{*}\right)$.

\section{A.7 Proof of Proposition 1}

Let $\Phi$ be a rule satisfying $\left(\mathbf{B I}^{*}\right),\left(\mathbf{S P}^{*}\right)$ and $\left(\right.$ Cont $\left.^{*}\right)$. First, we note that $\Phi$ satisfies a weak form of neutrality: if all candidates receive the same evaluation vector, they must all be elected. Consider a profile $m$ such that $\forall x \in X, m^{x}=u \in[0,1]^{I}$. Take $x \in X$, and consider the profile $n(\varepsilon)$ defined for $\varepsilon>0$ by $n(\varepsilon)^{x}=u$ and $\forall y \neq x, n(\varepsilon)^{y}=(1-\varepsilon) \cdot u$. By axiom (SP*), we have that $\Phi(n(\varepsilon))=\{x\}$. By axiom (Cont*), when $\varepsilon$ tends to 0 , we obtain that $x \in \Phi(m)$. We conclude that $\Phi(m)=X$.

Second, we show that if two candidates have the same evaluation vector, they must have the same fate: if $m^{x}=m^{y}$, then $x \in \Phi(m) \Rightarrow y \in \Phi(m)$. Assume $m^{x}=m^{y}=u \in[0,1]^{I}$ and suppose $x \in \Phi(m)$. Consider the profile $n$ defined by $\forall z \in X, n^{z}=u$, for which we just showed that $\Phi(n)=X$. We have $m^{x}=n^{x}, m^{y}=n^{y}, x \in \Phi(m)$ and $y \in \Phi(n)$. By application of (BI*), we obtain $y \in \Phi(m)$.

Third, we show that if a profile $n$ is obtained from a profile $m$ by replacing the evaluation vector of a candidate $y$ by the evaluation vector of a winner $x$ (in profile $m$ ), then $y$ must be a winner in profile $n$ : if $x \in \Phi(m), n^{y}=m^{x}$ and $\forall z \neq y, n^{z}=m^{z}$, then $y \in \Phi(n)$. To prove this claim, let $t \in \Phi(n)$. If $t=x$, we have $x \in \Phi(n)$, and therefore $y \in \Phi(n)$ by the second observation. If $t \neq x, y$, by application of ( $\left.\mathbf{B I}^{*}\right)$, we get that $x \in \Phi(n)$, and thus also $y \in \Phi(n)$.

Fourth, we show that if a profile $n$ is obtained from a profile $m$ by replacing the evaluation vector of a candidate $y$ by the evaluation vector of another candidate $z$, winners in $m$ (other 
than $y$ ) must remain winners in $n$ : if $x \in \Phi(m), n^{y}=m^{z}$ and $\forall t \neq y, n^{t}=m^{t}$, then $x \in \Phi(n)$. To prove this claim, let $t \in \Phi(n)$. If $t \neq y$, by application of $\left(\mathbf{B I}^{*}\right)$, we obtain that $x \in \Phi(n)$. If $t=y$, we know from our second observation that, as $n^{z}=n^{y}, z \in \Phi(n)$, and therefore $x \in \Phi(n)$.

Fifth, let us show that $z \in \Phi(m) \Leftrightarrow \sigma(z) \in \Phi\left(m^{\sigma}\right)$ when $\sigma$ is a transposition. Let $\sigma$ be a transposition switching $x$ and $y$, let $m \in M$ and let $z \in \Phi(m)$. To show that $\sigma(z) \in \Phi\left(m^{\sigma}\right)$, we consider two cases:

- $z \notin\{x, y\}$. In that case, $\sigma(z)=z$. Let $t \in \Phi\left(m^{\sigma}\right)$, and consider two sub-cases:

- $t \notin\{x, y\}$. By application of axiom (BI*) to profiles $m$ and $m^{\sigma}$, we obtain $z \in$ $\Phi\left(m^{\sigma}\right)$.

$-t \in\{x, y\}$, for instance (w.l.o.g.) $t=x$. Consider the profile $n$ defined by $n^{x}=$ $m^{y}, n^{y}=m^{y}$ and $\forall r \neq x, y, n^{r}=m^{r}$. As $z \in \Phi(m)$, we obtain from our fourth observation that $z \in \Phi(n)$. By application of (BI*) to profiles $n$ and $m^{\sigma}$, we get $z \in \Phi\left(m^{\sigma}\right)$.

- $z \in\{x, y\}$, for instance (w.l.o.g.) $z=x$, so that $\sigma(z)=y$. Let $t \in \Phi\left(m^{\sigma}\right)$, and consider two sub-cases:

- $t \notin\{x, y\}$. Consider the profile $n$ defined by $n^{x}=m^{x}, n^{y}=m^{x}$ and $\forall r \neq x, y, n^{r}=$ $m^{r}$. By our third observation, as $z=x \in \Phi(m)$, we get $y \in \Phi(n)$. Then, applying (BI*) to profiles $n$ and $m^{\sigma}$, we get $y \in \Phi\left(m^{\sigma}\right)$.

$-t \in\{x, y\}$. If $t=y$, the result is obtained directly. We are left to treat the remaining case: $z=t=x$. As $\# X \geq 3$, let $r \notin\{x, y\}$. We construct three profiles $m^{1}, m^{2}, m^{3}$, with $\left(m^{1}\right)^{x}=m^{x},\left(m^{2}\right)^{x}=\left(m^{3}\right)^{x}=m^{y} ;\left(m^{1}\right)^{y}=\left(m^{2}\right)^{y}=m^{y},\left(m^{3}\right)^{y}=m^{x}$; $\left(m^{1}\right)^{r}=\left(m^{2}\right)^{r}=\left(m^{3}\right)^{r}=m^{x}$ and $\forall s \neq x, y, r,\left(m^{1}\right)^{s}=\left(m^{2}\right)^{s}=\left(m^{3}\right)^{s}=m^{s}$. By our third observation, we get $r \in \Phi\left(m^{1}\right)$. By application of our fourth observation to $m^{1}$ and $m^{2}$, we get $r \in \Phi\left(m^{2}\right)$. By application of our third observation to $m^{2}$ and $m^{3}$, we get $y \in \Phi\left(m^{3}\right)$. By application of (BI*) to $m^{3}$ and $m^{\sigma}$, we get $y \in \Phi\left(m^{\sigma}\right)$. 
Finally, we conclude that, as any permutation is a product of transpositions, the rule $\Phi$ satisfies neutrality $\left(\mathrm{N}^{*}\right)$.

\section{A.8 Theorem 7: independence of the axioms}

Let us define the rule $\Phi^{3}$ by

$$
\Phi^{3}(m)=\arg \max _{x \in X} \sum_{i \in I} h^{x}\left(m_{i}\right)
$$

where $h^{x}\left(m_{i}\right)=\frac{1}{2} m_{i}^{x}+\frac{1}{5}\left(\min _{y \neq x} m_{i}^{y}+\max _{y \neq x} m_{i}^{y}\right)$. With this rule the value of an evaluation is a weighted sum of this evaluation and the lowest and highest evaluations given by the same voter to the other candidates. The weights are chosen so that $\Phi^{3}$ satisfies $\left(\mathbf{S P}^{*}\right)$ : indeed $m_{i}^{x}=m_{i}^{y} \Leftrightarrow$ $h^{x}\left(m_{i}\right)=h^{y}\left(m_{i}\right)$ and $m_{i}^{x}>m_{i}^{y} \Leftrightarrow h^{x}\left(m_{i}\right)>h^{y}\left(m_{i}\right)$. We observe that $\Phi^{3}$ also satisfies $\left(\mathbf{A}^{*}\right)$, (Cont*), (Sep*) and (CI*). The rule $\Phi^{3}$ also satisfies (SI*) since $h^{x}\left(\mathbb{1}-m_{i}\right)=\frac{9}{10}-h^{x}\left(m_{i}\right)$. Moreover, $\Phi^{3}$ fails to satisfy $\left(\mathbf{B I}^{*}\right)$. Consider the profile $m$ such that $m_{1}=(0,1,0,1)$ and $m_{2}=(1,0,0,0)$ and profile $n$ such that $n_{1}=(0,1,0,0)$ and $n_{2}=(1,0,0,0)$ (candidates are ranged in the following order: $x, y, z, t$, voters are 1,2$)$. We have $n^{x}=m^{x}$ and $n^{y}=m^{y}$. Moreover, $\Phi^{3}(m)=\{y, t\}$ and $\Phi^{3}(n)=\{x, y\}$, therefore $\Phi^{3}$ violates (BI*).

\section{A.9 Proof of Proposition 2}

The following example is adapted from Sertel and Slinko (2007). Take $\Gamma=\{A, B, C, D\}$, with the order $A \succ_{\Gamma} B \succ_{\Gamma} C \succ_{\Gamma} D$, and assume $\# I=3$. Consider the following anonymous rule: $A^{3} \succ A^{2} B \succ A^{2} C \succ A^{2} D \succ A B^{2} \succ A B C \succ A B D \succ A C^{2} \succ A C D \succ B^{3} \succ B^{2} C \succ A D^{2} \succ$ $B^{2} D \succ B C^{2} \succ B C D \succ B D^{2} \succ C^{3} \succ C^{2} D \succ C D^{2} \succ D^{3}$, where $B^{2} C \succ A D^{2}$ means that $(B, B, C) \succ(A, D, D)$. The rule satisfies (SP) and (Sep), as can be verified. However, we have: (a) $A C D \succ B^{3}$; (b) $B^{2} C \succ A D^{2}$; and (c) $B D^{2} \succ C^{2} D$.

Assume that the rule is additively separable, with value $f$. We have then: (a) $f(A)+f(C)+$ $f(D)>3 f(B) ;($ b) $2 f(B)+f(C)>f(A)+2 f(D)$; and (c) $f(B)+2 f(D)>2 f(C)+f(D)$. We obtain by summation: $f(A)+3 f(B)+2 f(C)+3 f(D)>f(A)+3 f(B)+2 f(C)+3 f(D)$, hence a contradiction. 
Remark (for Section 6.1): the rule $\succeq$ introduced above cannot be extended into a (anonymous) variable-population rule satisfying Reinforcement. If this was the case, we would have $A C D B^{2} C \succ B^{3} A D^{2}$ (from (a) and (b)). And then $A C D B^{2} C B D^{2} \succ B^{3} A D^{2} C^{2} D$ (combining with (c)). This would violate the anonymity of the variable-population rule.

\section{References}

Alcantud, J. C. R. And A. Laruelle (2013): "Dis\&approval voting: a characterization," Social Choice and Welfare, 41, 1-10.

Aleskerov, F., V. V. Chistyakov, and V. Kalyagin (2010): "The threshold aggregation," Economics Letters, 107, 261-262.

Alós-Ferrer, C. (2006): "A simple characterization of approval voting," Social Choice and Welfare, 27, 621-625.

BALINSKI, M. L. AND R. LARAKI (2011): Majority judgment: measuring, ranking, and electing, The MIT Press.

Baujard, A., H. Igersheim, F. Gavrel, J.-F. Laslier, And I. Lebon (2016): "Individual behavior under Evaluative Voting. A comparison between laboratory and in situ experiments," in Voting Experiments, Springer.

Brams, S. J. And P. C. Fishburn (1978): "Approval voting," The American Political Science Review, 72, 831-847.

D'Aspremont, C. And L. Gevers (2002): "Social welfare functionals and interpersonal comparability," in Handbook of social choice and welfare, Elsevier, vol. 1, 459-541.

Debreu, G. (1960): "Topological methods in cardinal utility theory," in Mathematical methods in the social sciences, Stanford University Press, 16-26.

Dhillon, A. And J.-F. Mertens (1999): "Relative utilitarianism," Econometrica, 67, 471498.

Gaertner, W. And Y. Xu (2012): "A general scoring rule," Mathematical Social Sciences, 63, 193-196.

Hillinger, C. (2005): "The case for utilitarian voting," Homo Oeconomicus, 23, 295-321.

Igersheim, H., A. Baujard, F. Gavrel, J.-F. Laslier, And I. Lebon (2016): "Individual Behavior Under Evaluative Voting: A Comparison Between Laboratory and In Situ Experiments," in Voting Experiments, Springer, 257-269.

Krantz, D. H., R. D. Luce, P. Suppes, And A. Tversky (1971): Foundations of Measurement. Vol. 1: Additive and Polynomial Representations, Academic Press, New York.

MACÉ, A. (2015): "Voting with evaluations: When should we sum? What should we sum?" Aix-Marseille School of Economics working paper. 
Maskin, E. (1978): "A theorem on utilitarianism," The Review of Economic Studies, 45, 93-96.

MAY, K. O. (1952): "A set of independent necessary and sufficient conditions for simple majority decision," Econometrica, 20, 680-684.

Moulin, H. (1991): Axioms of cooperative decision making, Cambridge University Press.

Myerson, R. B. (1995): "Axiomatic derivation of scoring rules without the ordering assumption," Social Choice and Welfare, 12, 59-74.

NÚÑEZ, M. AND J.-F. LASLIER (2014): "Preference intensity representation: strategic overstating in large elections," Social Choice and Welfare, 42, 313-340.

OK, E. A. (2007): Real analysis with economic applications, Princeton University Press.

Pivato, M. (2013a): "Formal utilitarianism and range voting," Mathematical Social Sciences, $67,50-56$. $210-221$.

Sertel, M. And A. Slinko (2007): "Ranking committees, income streams or multisets," Economic Theory, 30, 265-287.

Sмiтh, J. H. (1973): "Aggregation of preferences with variable electorate," Econometrica, 41, $1027-1041$.

Smith, W. D. (2000): "Range voting," Mimeo.

Sprumont, Y. (2016): "Ranking by rating," Theoretical Economics.

Thomson, W. (2003): "Axiomatic and game-theoretic analysis of bankruptcy and taxation problems: a survey," Mathematical Social Sciences, 45, 249-297.

Young, H. P. (1974): "An axiomatization of Borda's rule," Journal of Economic Theory, 9, $43-52$.

(1975): "Social choice scoring functions," SIAM Journal on Applied Mathematics, 28, $824-838$. 